\title{
5 GHz band low phase noise Si-CMOS oscillator using FBAR
}

\author{
Shoichi Tanifujia), Tuan Thanh Ta, Suguru Kameda, \\ Tadashi Takagi, and Kazuo Tsubouchi \\ Research Institute of Electrical Communication, Tohoku University, \\ 2-1-1 Katahira, Aoba-ku, Sendai, Miyagi, 980-8577 Japan \\ a) tanifuji@riec.tohoku.ac.jp
}

Abstract: In this paper, low phase noise $5 \mathrm{GHz}$ oscillator is presented. This oscillators designed with $90 \mathrm{~nm}$ silicon complementary metal oxide semiconductor (Si-CMOS) process. To achieve low phase noise, we used high $Q$ value film bulk acoustic resonator (FBAR) instead of conventional $L C$ resonant circuit. This FBAR oscillator has phase noise of lower than $-130 \mathrm{dBc} / \mathrm{Hz}$ at $1 \mathrm{MHz}$ offset.

Keywords: film bulk acoustic resonator (FBAR), $5 \mathrm{GHz}, 90 \mathrm{~nm}$ silicon complementary metal oxide semiconductor (Si-CMOS), low phase noise, oscillator

Classification: Electron devices, circuits, and systems

\section{References}

[1] A. Hajimiri and T. H. Lee, "A general theory of phase noise in electrical oscillators," IEEE J. Solid-State Circuits, vol. 33, no. 2, pp. 179-194, Feb. 1998.

[2] N. Itoh, "A study of capacitor-coupled varactor VCO to investigate flicker noise up-conversion mechanism," IEEE International Workshop on Radio-Frequency Integeration Technology (RFIT 2005), pp. 157-160, Nov. 2005.

[3] D. B. Lesson, "A simple model of feedback oscillator noise spectrum," IEEE Proc., vol. 54, no. 2, pp. 329-330, Feb. 1966.

[4] Y. Ito, H. Sugawara, K. Okada, and K. Masu, "A 0.98 to $6.6 \mathrm{GHz}$ tunable wideband VCO in a 180nm CMOS technology for reconfigurable radio transceiver," IEEE Asian Solid-State Circuits Conference (ASSCC 2006), pp. 359-362, Nov. 2006.

[5] K. Nakamura, H. Sasaki, and H. Shimizu, "ZnO/SiO ${ }_{2}$-diaphragm composite resonator on a silicon wafer," Electron. Lett., vol. 17, no. 14, pp. 507-509, July 1981.

[6] T. W. Grudkowski, J. F. Black, T. M. Reeder, D. E. Cullen, and R. A. Wagner, "Fundamental-mode VHF-UHF miniature acoustic resonators and filters on silicon," Appl. Phys. Lett., vol. 37, no. 11, pp. 993-995, Dec. 1980 .

[7] K. M. Lakin and J. S. Wang, "Acoustic wave bulk composite resonators," Appl. Phys. Lett., vol. 38, no. 3, pp. 125-127, Feb. 1981.

[8] K. Tsubouchi and N. Mikoshiba, "Zero-temperature-coefficient SAW devices AlN epitaxial films," IEEE Trans. Sonics Ultrason., vol. 32, no. 5, 
pp. 634-644, Sept. 1985.

[9] H. Zhang, J. Kim, W. Pang, H. Yu, and E. S. Kim, "5GHz low-phasenoise oscillator based on FBAR with low TCF," IEEE Int. Conf. SolidState Sensors, Actuators and Microsystems, vol. 1, pp. 1100-1101, June 2005.

[10] M. Aissi, E. Tournier, M. A. Dubois, G. Parat, and R. Plana, "A 5.4GHz $0.35 \mu \mathrm{m}$ BiCMOS FBAR resonator oscillator in above-IC technology," IEEE Int. Solid-State Circuits Confrence 2006 (ISSCC 2006), pp. 12281235, Feb. 2006.

\section{Introduction}

Low phase noise oscillators are one of the most important components of radio transceivers, since their performance set the limits on the dynamic range and jamming sensitivity of the transceivers. Recently, to create small and low cost transceivers, fully integrated oscillators using silicon complementary metal oxide semiconductor (Si-CMOS) process has been developed $[1,2,3,4]$. But because of noisy Si-CMOS transistor and low $Q$ value inductor due to lossy low resistivity Si substrate, Si-CMOS based oscillators have poorer phase noise characteristic than that of compound semiconductors based ones. It is necessary to lower phase noise of Si-CMOS oscillators. In this paper, to improve the phase noise of oscillators, we used film bulk acoustic resonator (FBAR) $[5,6,7]$ instead of conventional $L C$ resonant circuit. FBAR have great advantages of high $Q$ value, low insertion loss and small size over $2 \mathrm{GHz}$. We have fabricated $5 \mathrm{GHz}$ band FBAR using aluminium nitride (AlN) film. AlN has high acoustic velocity of $1.1 \times 10^{4} \mathrm{~m} / \mathrm{s}$ and good temperature coefficient of frequency $(T C F)$ of $25 \mathrm{ppm} /{ }^{\circ} \mathrm{C}[8]$. Therefore, we can obtain low phase noise oscillator by high $Q$ value FBAR, more over FBAR oscillator can be realized standard oscillator by good $T C F$. In this paper, we improved phase noise using FBAR.

\section{Device Fabrication}

(a) FBAR Fabrication

FBAR structure has piezoelectric film sandwiched between bottom and top electrodes. We used membrane structure of $\mathrm{Pt} / \mathrm{ZrO}_{2} / \mathrm{SiO}_{2} / \mathrm{Si}(100)$. Resistance of $\mathrm{Si}(100)$ was a high value of more than $1 \mathrm{k} \Omega \mathrm{cm}$. $\mathrm{SiO}_{2}$ was thermal oxidization method on $\mathrm{Si}(100)$ to stop $\mathrm{Si}$ etching. We have selected $\mathrm{Pt} / \mathrm{ZrO}_{2}$ structure for bottom electrodes of FBAR. Pt has suitable characteristics to obtain high oriented AlN film. Thin $\mathrm{ZrO}_{2}$ layer was used as a bonding layer between $\mathrm{SiO}_{2} / \mathrm{Si}(100)$ substrate and Pt layer. $\mathrm{ZrO}_{2}$ and $\mathrm{Pt}$ layers were deposited by RF magnetron sputtering method. Thickness of $\mathrm{ZrO}_{2}$ and Pt were $10 \mathrm{~nm}$ and $50 \mathrm{~nm}$, respectively.

We have successfully grown $c$-axis oriented AlN film deposited by electron cyclotron resonance (ECR) plasma sputter method on $\mathrm{Pt} / \mathrm{ZrO}_{2} / \mathrm{SiO}_{2} / \mathrm{Si}$ substrate. Full width at half maximum $(F W H M)$ of $\mathrm{AlN}(0002)$ on $\mathrm{Pt} / \mathrm{ZrO}_{2}$ 


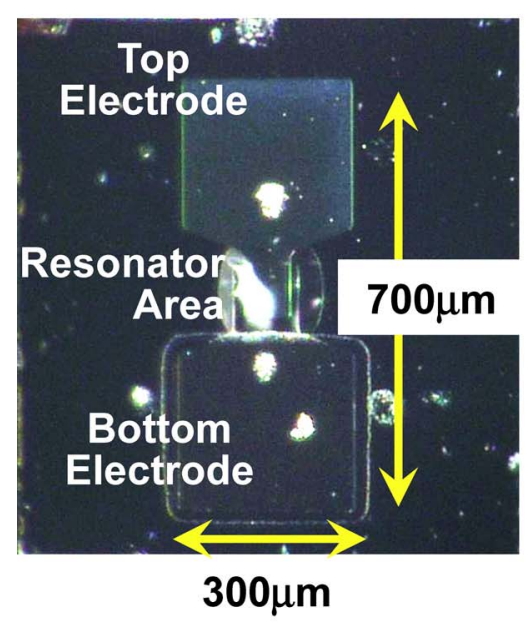

(a)

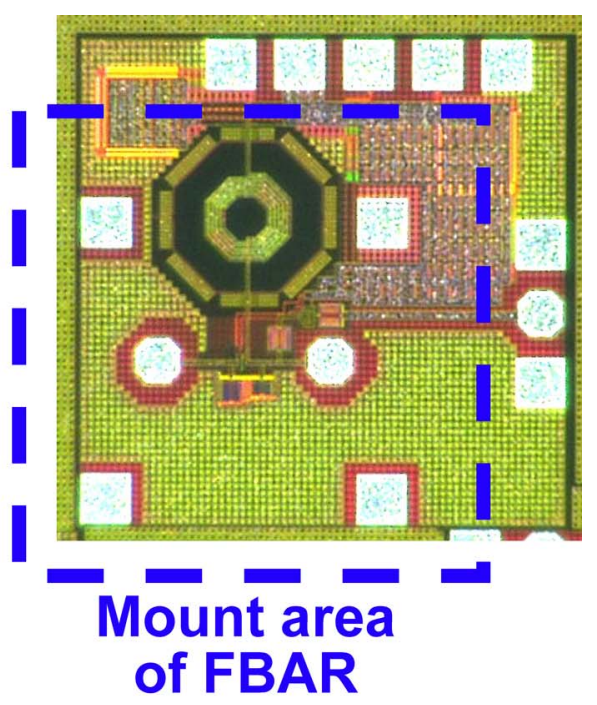

(b)

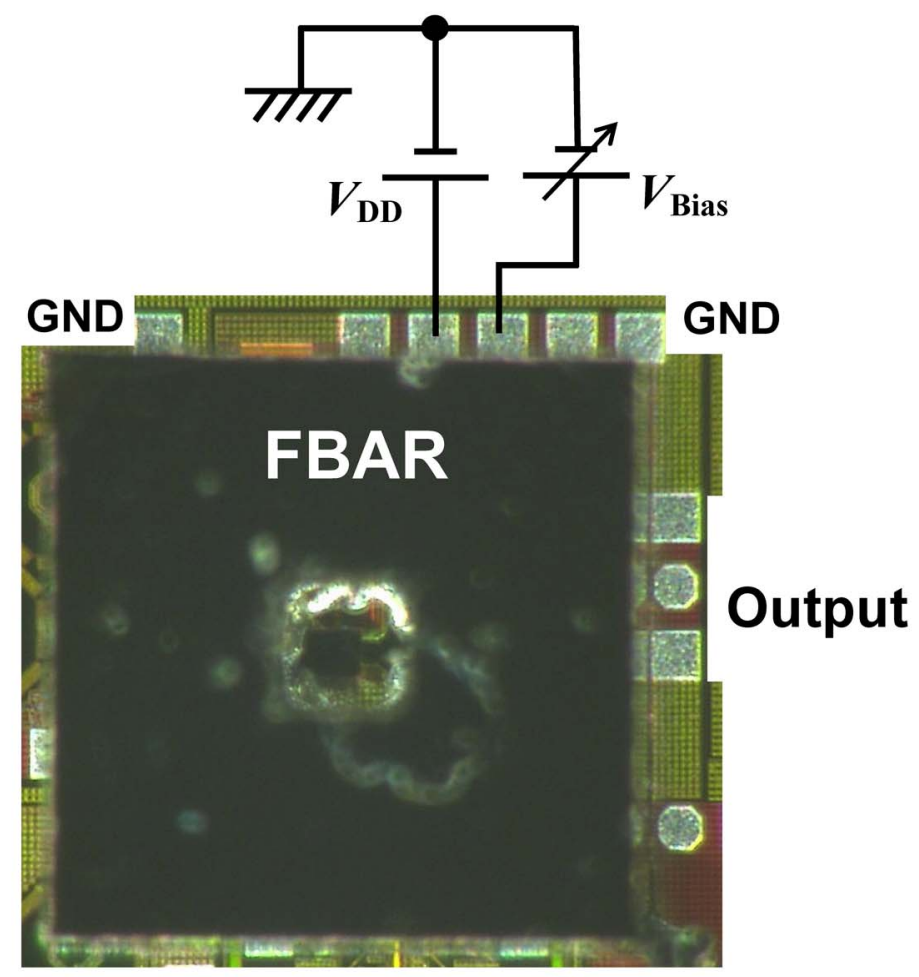

(c)

Fig. 1. Chip micrograph of (a) FBAR, (b) Oscillator circuit and (c) Oscillator chip mounted FBAR.

bottom electrode was excellent value of $1.3^{\circ}$. AlN thickness was $580 \mathrm{~nm}$. Top electrode materials was Ag deposited by RF magnetron sputtering method. $\mathrm{Ag}$ thickness was $60 \mathrm{~nm}$. Finally, we formed cavity under membrane structure used by deep-reactive ion etching (D-RIE) method.

(b) Oscillator design

Oscillator was basic colpitts circuit and it designed by using $90 \mathrm{~nm} \mathrm{Si-}$ CMOS process of Taiwan Semiconductor Manufacturing Company, Limited 


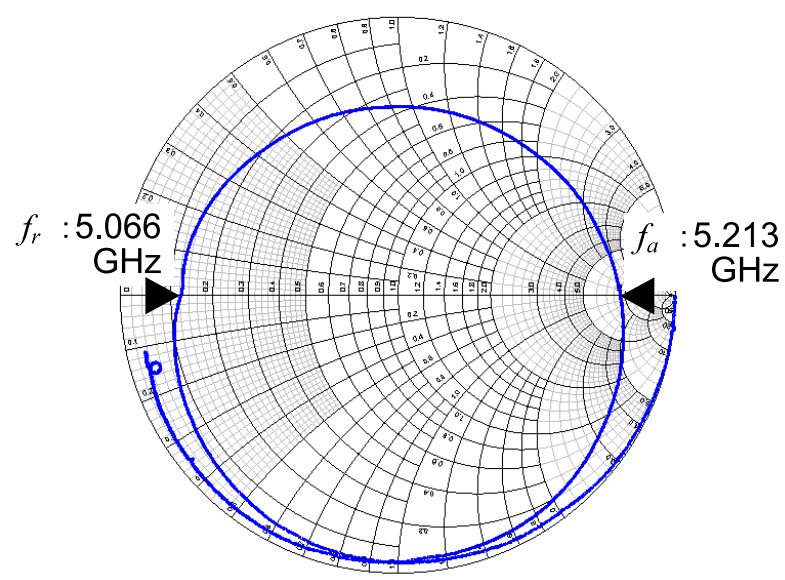

(a)

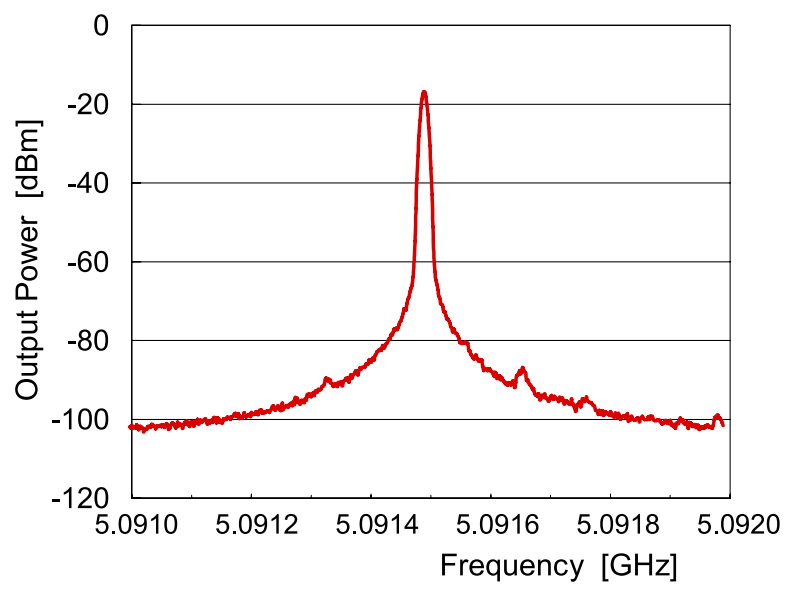

(b)

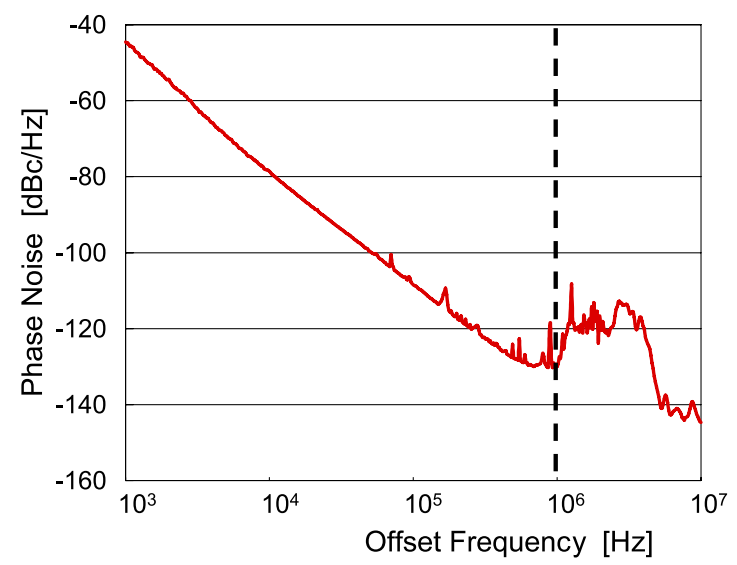

(c)

Fig. 2. Output characteristics of (a) FBAR resonant response $\left(S_{11}\right)$, (b) Power spectrum and (c) Phase noise.

(TSMC). FBAR was mounted by flip-chip mounting using stud bump bonding (SBB) method on IC chip. Figure 1 shows Chip micrograph of FBAR and oscillator. The FBAR was die size of $1 \times 1 \mathrm{~mm}^{2}$. The oscillator circuit 
Table I. Performance summary and comparison with other works.

\begin{tabular}{|c||c|c|c|}
\hline & This work & Ref. [9] & Ref. [10] \\
\hline \hline Oscillation Frequency & $5 \mathrm{GHz}$ & $5 \mathrm{GHz}$ & $5.4 \mathrm{GHz}$ \\
\hline Process & $90 \mathrm{~nm}$ & Discrete & $0.35 \mu \mathrm{m} \mathrm{SiGe}$ \\
technology & Si-CMOS & BJT & BiCMOS BJT \\
\hline Phase noise (@100 kHz) & $-108.4 \mathrm{dBc} / \mathrm{Hz}$ & $-109.5 \mathrm{dBc} / \mathrm{Hz}$ & $-117.7 \mathrm{dBc} / \mathrm{Hz}$ \\
\hline
\end{tabular}

was also $1 \times 1 \mathrm{~mm}^{2}$.

\section{Experimental Results}

We measured oscillation frequency and output power on-wafer by using Series Spectrum Analyzer E4440A (Agilent Technologies, Inc.). Figure 2 (a) shows resonant characteristics of FBAR $\left(S_{11}\right)$. Resonant frequency $\left(f_{r}\right)$ was $5.066 \mathrm{GHz}$. Anti-resonant frequency $\left(f_{a}\right)$ was $5.213 \mathrm{GHz}$. $Q$ value and effective electromechanical coupling coefficient $\left(k_{\text {eff }}^{2}\right)$ were 819 and $7.1 \%$, respectively. Figure 2 (b) shows output characteristics of power spectrum. Horizontal axis is frequency and vertical axis is output power. Oscillating frequency was $5.0909 \mathrm{GHz}$, and it does not show any change during measurement. Figure 2 (c) shows phase noise characteristics measured by using Signal Source Analyzer E5052A (Agilent Technologies, Inc.). Horizontal axis is offset frequency and vertical axis is phase noise. Phase noise of designed FBAR oscillator is lower than $-130 \mathrm{dBc} / \mathrm{Hz}$ at $1 \mathrm{MHz}$ offset.

Table I shows performance summary and comparison with other works of FBAR oscillators. Their oscillator is using FBAR and oscillation frequency is $5 \mathrm{GHz}$ band. Though, our work was Si-CMOS process with noisy transister, but we obtained near phase noise level of BiCMOS process. Those were results using nano fabrication technology of $90 \mathrm{~nm}$ process and high $Q$ value FBAR.

\section{Conclusion}

Low phase noise oscillator was proposed by using film bulk acoustic resonator (FBAR). Oscillator chip was realized using $90 \mathrm{~nm}$ silicon complementary metal oxide semiconductor (Si-CMOS) process with small die size of $1 \times 1 \mathrm{~mm}^{2}$. FBAR was mounted by flip-chip mounting using stud bump bonding (SBB) method on oscillator IC chip. FBAR oscillator has low phase noise at $1 \mathrm{MHz}$ offset of lower than $-130 \mathrm{dBc} / \mathrm{Hz}$ at $5 \mathrm{GHz}$ band.

\section{Acknowledgments}

The authors would like to thank Core Research for Evolutional Science and Technology (CREST) program of the Japanese Science and Technology Agency (JST) for fund support. 